\title{
Superimposed Non-acid Reflux Event: An Example of When It May Be Important to Revisit the Impedance Analysis Guidelines
}

TO THE EDITOR: Combined multichannel intraluminal impedance and esophageal $\mathrm{pH}(\mathrm{MII}-\mathrm{pH})$ monitoring is the preferred method for assessing gastroesophageal reflux (GER) because it permits (1) detection and analysis of both acid $(\mathrm{pH}<4.0)$ and non-acid ( $\mathrm{pH} \geq 4.0$ ) GER, and (2) monitoring of proximal extent of individual GER events. ${ }^{1}$ The duration of a GER episode is always assessed in the distal-most impedance channel (Z6) because total bolus exposure is greatest nearest the lower esophageal sphincter. By definition, a GER episode begins when impedance drops to $50 \%$ of baseline ( $\mathrm{Z} 6)$ and is cleared when impedance again ascends to $\geq 50 \%$ of baseline (Z6). By convention, an impedance-detected event is not counted if the duration is not $\geq 5$ seconds. ${ }^{2,3}$ MII-pH software uses these guidelines to run preliminary GER autoscans to reduce observer burden. Due to low autoscan specificity, expert opinion has recommended against analysis of MII-pH tracings using only the autoscan feature for infants and children. ${ }^{4}$ They also suggest that automated GER detection needs to be refined by consensus in order to derive meaningful autoscan results. ${ }^{4}$ It is important to note that automated analysis overestimates the frequency of reflux events, in particular, non-acid GER events. ${ }^{4-7}$

Occasionally, the MII-pH autoscan will mark an event that, on first glance, appears to be a legitimate single reflux event (for example, Figure A) but upon further inspection, it becomes clear that the software algorithm actually combined 2 events. This happens when the impedance waveform in the distal channel of the first event does not reach $50 \%$ of baseline before the overlapping second event enters the esophagus (Figure B). In this particular example, separation results in 2 GER events with durations that are both less than 5 seconds (3.9 seconds and 4.3 seconds). Figure $\mathrm{C}$ depicts a contour plot that confirms the conjoined/superimposed event. This image was captured as part of an assessment of a 3-week-old male who was referred to our facility for symptoms suggestive of GER.
Loots and colleagues ${ }^{4}$ reported that the majority of MII-pH observers in their inter- and intra-observer study indicated that they tag GER events that fail to meet accepted guidelines because they felt that the guidelines were inadequate. While the specific guidelines that were ignored were not detailed, it is likely that these would include (1) minimum duration of 5 seconds, and (2) the requirement for the impedance waveform in the $\mathrm{Z} 6$ channel to reach $50 \%$ of baseline to signal the end of bolus clearance. There are likely many instances wherein an observer who frequently assesses MII-pH tracings will encounter a GER episode during which the duration in the distal channel is less than 5 seconds, and/or the baseline impedance is low and the $50 \%$ baseline target is not achieved. Because determining the frequency of GER is an important component of the MII-pH report, the event described here provides an example of where revisiting the impedance tracing analysis guidelines to include GER events wherein (1) bolus duration is < 5 seconds, and (2) the end of bolus clearance is not $50 \%$ of baseline.

Our experience has been that when one of these conjoined/ superimposed events is encountered in a tracing, the observer can usually expect to see others. To our knowledge, this is the first time that a non-acid re-reflux/superimposed event has ever been reported. Previous reports have described re-reflux/superimposed events as being associated only with acid reflux. ${ }^{2,8-10}$ Additional investigation is needed to further examine the potential clinical value of these events as they may relate to the pathogenesis of GER disease in infants, in particular, who experience large numbers of non-acid GER events and have underdeveloped reflux barriers.

Frederick W Woodley ${ }^{1,2,3}$

${ }^{1}$ Center for Motility Disorders, Nationwide Children's Hospital,

Columbus, OH, USA ${ }^{2}$ Division of Gastroenterology, Hepatology and Nutrition, Nationwide Children's Hospital, Columbus, OH, USA; and ${ }^{3}$ Department of Pediatrics, The Ohio State University College of 

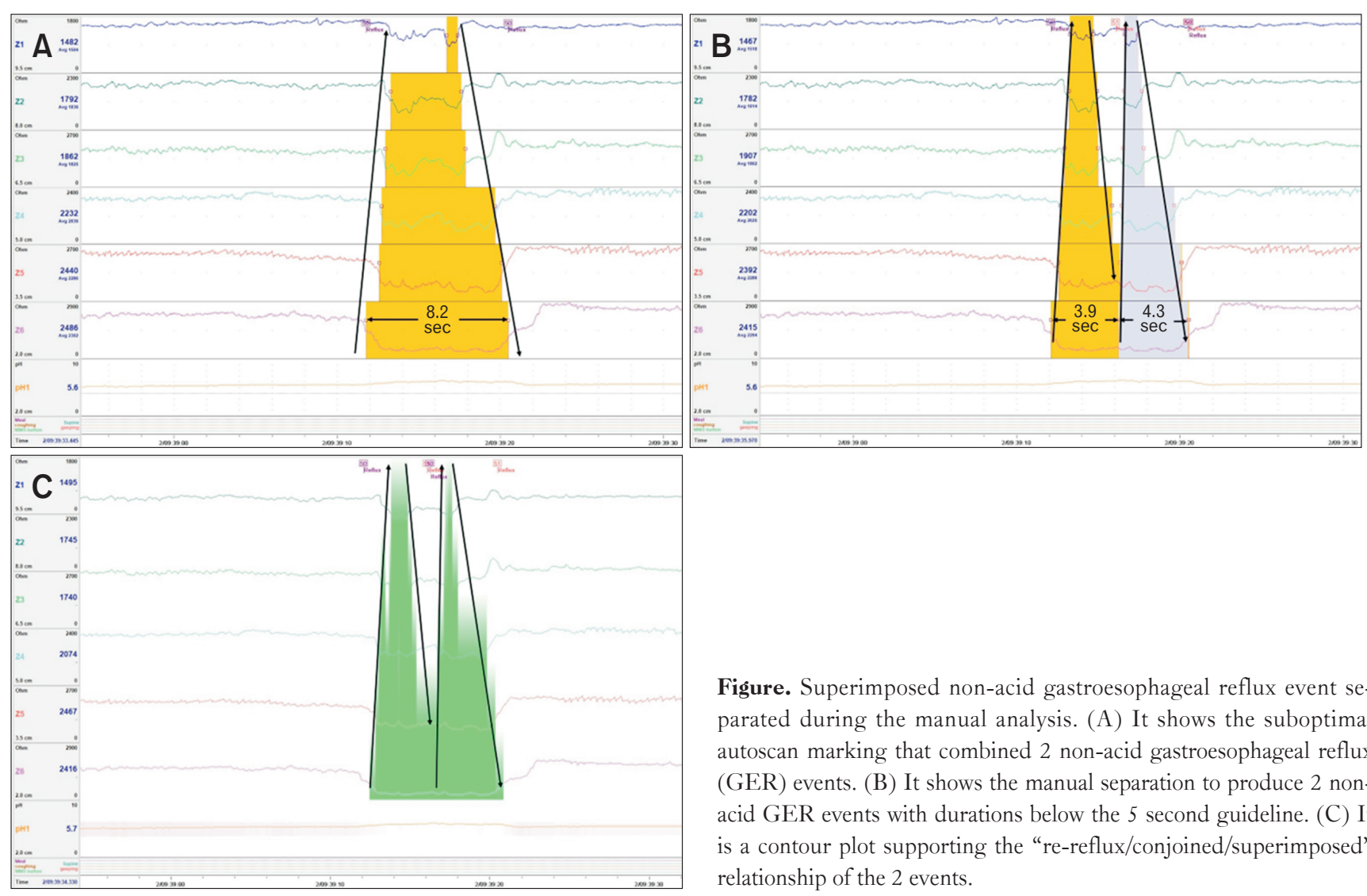

Figure. Superimposed non-acid gastroesophageal reflux event separated during the manual analysis. (A) It shows the suboptimal autoscan marking that combined 2 non-acid gastroesophageal reflux (GER) events. (B) It shows the manual separation to produce 2 nonacid GER events with durations below the 5 second guideline. (C) It is a contour plot supporting the "re-reflux/conjoined/superimposed" relationship of the 2 events.

1. Safe M, Cho J, Krishnan U. Combined multichannel intraluminal impedance and $\mathrm{pH}$ measurement in detecting gastroesophageal reflux disease in children. J Pediatr Gastroenterol Nutr 2016;63:e98-e106.

2. Shay S, Tutuian R, Sifrim D, et al. Twenty-four hour ambulatory simultaneous impedance and $\mathrm{pH}$ monitoring: a multicenter report of normal values from 60 healthy volunteers. Am J Gastroenterol 2004;99:10371043.

3. Skopnik H, Silny J, Heiber O, Schulz J, Rau G, Heimann G. Gastroesophageal reflux in infants: evaluation of a new intraluminal impedance technique. J Pediatr Gastroenterol Nutr 1996;23:591-598.

4. Loots CM, van Wijk MP, Blondeau $\mathrm{K}$, et al. Interobserver and intraobserver variability in $\mathrm{pH}$-impedance analysis between 10 experts and automated analysis. J Pediatr 2012;160:441-446, e1.

5. Wenzl TG, Benninga MA, Loots CM, Salvatore S, Vandenplas Y; ESPGHAN EURO_PIG Working Group. Indications, methodology, and interpretation of combined esophageal impedance-pH monitoring in children: ESPGHAN EURO-PIG standard protocol. J Pediatr Gastroenterol Nutr 2012;55:230-234.

6. Ravi K, DeVault KR, Murray JA, Bouras EP, Francis DL. Inter-observer agreement for multichannel intraluminal impedance-pH testing. Dis Esophagus 2010;23:540-544.

7. Roman S, Bruley des Varannes S, Pouderoux P, et al. Ambulatory 24-h oesophageal impedance-pH recordings: reliability of automatic analysis for gastro-oesophageal reflux assessment. Neurogastroenterol Motil 2006;18:978-986.

8. Shay SS, Johnson LF, Richter JE. Acid rereflux: a review, emphasizing detection by impedance, manometry, and scintigraphy, and the impact on acid clearing pathophysiology as well as interpreting the $\mathrm{pH}$ record. Dig Dis Sci 2003;48:1-9.

9. Sifrim D, Castell D, Dent J, Kahrilas PJ. Gastro-oesophageal reflux monitoring: review and consensus report on detection and definitions of acid, non-acid, and gas reflux. Gut 2004;53:1024-1031.

10. Shin MS. Esophageal $\mathrm{pH}$ and combined impedance-pH monitoring in children. Pediatr Gastroenterol Hepatol Nutr 2014;17:13-22.

Acknowledgements: The author would like to thank Jason Schmidt and Peter Lu MD at Nationwide Children's Hospital for technical assistance and review of the manuscript, respectively, and to Rodrigo Strehl Machado MD-PhD at the Universidad de Sao Paulo for review of the manuscript.

Financial support: None.

Conflicts of interest: None. 